\title{
An Analysis of Link Disjoint and Node Disjoint Multipath Routing for Mobile Ad Hoc Network
}

\author{
Indrani Das \\ School of Computer and Systems Sciences, Jawaharlal Nehru University, New Delhi, 110067, India \\ E-mail: indranidas2000@gmail.com \\ D.K. Lobiyal and C.P.Katti \\ School of Computer and Systems Sciences, Jawaharlal Nehru University, New Delhi, 110067, India \\ E-mail: lobiyal@gmail.com, cpkatti@yahoo.com
}

\begin{abstract}
In Mobile Ad hoc Network, path between source and destination node changes too frequently due to unpredictable behavior and movement of mobile nodes. The data delivery to the intended destination becomes very challenging. The paths exist between source and destination node may be various types. Data delivery may be done with single or multiple paths. Single path sometimes not guaranteed about data delivery, so one of the better solution is multipath data delivery. Here, in this paper, we have considered Link Disjoint and Node Disjoint multipath for data delivery. For this AOMDV protocol with node and link disjoint is considered to evaluate performance. To evaluate their performance different node pause time considered with varying number of nodes. We have computed various QoS network metrics like throughput, average end-to-end delay, routing overhead to identify in which method of data delivery perform better and in what conditions. The results obtain shows that Node Disjoint multipath method AOMDV performs better than Link Disjoint method. The simulation work carried out using Simulator NS-2.34.
\end{abstract}

Index Terms - Link-Disjoint, Node-Disjoint, Throughput, end-to-end delay, routing overhead, pause time.

\section{INTRODUCTION}

A Mobile Ad hoc Network is a collection mobile device that can communicate with each other without using any fixed network infrastructure through wireless medium [1]. Mobile nodes can communicate with each other directly or through intermediate nodes and form a multi hop communication. If nodes are fall in each other transmission coverage can communicate without intermediate node. The challenging characteristics of this network are: frequent changes in the network topology, frequent link breakage. This is occurs in the network due to depleted battery power may leads to mobile nodes are die in the network or mobile nodes frequently leave the coverage area from each other. In this situation data delivery between source and destination node with a single path is very difficult or sometimes it may not be possible. The packet drop is very high in such network scenarios. To cope up from such situation multipath data delivery is very effective. In multipath routing more than one path discovered between source and destination node. Data packet can be send concurrently in all path or data packet can be send by selecting path one after another. Routing algorithm need to select path tactfully to deliver data efficiently $[6,7]$. To deliver data packets through node disjoint and link disjoint path may be one way to achieve fault tolerant path. This is one of the major advantages of using multipath routing data delivery. If some path is fails still there are other paths available to cope with failure. The application of such network is very wide like battle field, disaster management, under water network etc.

In this paper, a variant of well-known AOMDV protocol node disjoint and link disjoint multipath routing is simulated and analyzed. We have computed various QoS matrices i.e. throughput, average end-to-end delay, routing overhead with variable node density and pause time. To the best of our knowledge and so far literature studied no such analysis is exist in the context of Mobile Ad Hoc Networks.

The rest of the paper is organized as follows. In section II we have discussed the works related to multipath routing protocols. Section III included brief discussion about node disjoint and link disjoint path and AOMDV protocol. Simulation work and results analysis is presented in Section IV and finally, we have concluded the work in Section V.

\section{RELATED WORKS}

Due to numerous benefits of multipath routing over single path data delivery has attracted lots of attention. The main target of multipath routing protocols is to reduces the use of scarce network resources i.e. battery power, bandwidth. Further, it may also reduce fault occurrence, minimize average end-to-end delay, distribute load or traffic to various paths and ensure the guarantee of message delivery. This way multipath routing protocol maintains various QoS parameters of any network. Here we have discussed some of the multipath routing protocols work on node disjoint and link disjoint multipath techniques.

Kejia Zhang et al. in [8] analyzed effect of disjoint 
paths exists between pair of sensor nodes which may increase the various network parameters like robustness, throughput, and load balancing. They proposed an efficient distributed algorithm known as DFDP (Distributedly finding disjoint paths) to find $k$ disjoint paths exist between pair of node. Natarajan Meghanathan in [9] proposed link-disjoint, node-disjoint and zonedisjoint multi-path algorithms focusing on the trade-off between lifetime and hop count of routes for MANET. Through these three techniques successive multipath discoveries and the number of disjoint paths, hop count per multipath are thoroughly analyzed. The number of zone disjoint paths per multipath is minimum compare to others. Also, time elapsed in path discoveries process zone disjoint techniques outperforms others. X. Huang et al. in [10] evaluated the applicability of node disjoint multipath in the context of VANETs and compare the same with single path. Through simulation results author's shows the node disjoint multipath techniques can be used in VANETs, because it improves the packet delivery ratio and reduces the delay in packet delivery. $\mathrm{W}$ Almobaideen et al. in [11] proposed a modified form of AOMDV known as Maximally Spatial Disjoint Multipath routing protocol (MSDM) for MANETs. The data delivered through multiple paths which are mostly spatially separated and node-disjoint paths. Results show that spatially node-disjoint routes perform better than AOMDV which selects only link-disjoint paths. Abhishek et al. [12] proposed NMN-AODV protocol which is based on AODV. In this protocol only two node-disjoint routes between source and destination node are established for data transmission. As compare to MPAODV this protocol uses minimum number of control packet exchange and establish node disjoint path faster than the MP-AODV. Resulting end-to-end delay is less than the MP-AODV. S. Waharte et al. [13] proposed a 2path routing protocol which is a special case of multipath routing. They have shown the benefits of spreading traffic over multiple paths to improve overall network performance. This protocol evaluated in a static and nonenergy-constrained network. One of the important parameter called interference among paths is bad effect the network performance. They also studied the effect of interference on single and multiple source-destination pair scenarios. In the presence of interference network capacity is degraded and voids the benefits of multipath routing. The 2-path protocol identifies two paths between source and destination. All the node participated in path-1 and path-2 form a connected graph. There is no edge shared between path-1 and path-2, which ensure that path discovered is free from interference. V. Kudachi et al. [14] proposed a node disjoint multipath routing protocol based on AODV. They addressed residual energy of nodes and discovery of multiple node disjoint paths with low routing overhead. M.Nagaratna et al. [15] addressed the frequent path breakage in MANET due to which network performance is not good. They have computed multiple paths between source and destination pair using node disjoint methodology. An improvement is shown when data delivered through multiple paths in term of reliability, Route Request Frequency and end-to-end delay. V.K. Barbudhe et al. [16] proposed a node disjoint multipath protocol based on AODV with additional features data security during transmission and link failure. This protocol reduces routing overhead significantly because through a single flooding of a RREQ message route discovery and maintenance process completes. S. Upadhayaya et al. [17] proposed a Node Disjoint Multipath Routing Considering Link and Node Stability (NDMLNR). They mainly focused on QoS routing in MANETs but no experimental analysis is discussed in the paper only theoretical analysis is presented. P.Periyasamy [21] presented a survey on three categories of multipath routing protocols along with their comparison. In [22] P.Periyasamy et al. compare the performance of various existing routing protocols like AOMDV, OLSR and ZRP with different network scenarios. The network scenarios are created from various well known mobility models. Also, they have evaluated these protocols based on two different traffic sources are TCP and CBR. In [23] authors address the security issues on AOMDV routing protocol. The simulation results shows security attacks effects the network throughput and delay but packet loss remain unaffected. Finally, security attacks need to be address with better counter measure for great applicability of multipath routing protocol. P.Periyasamy et al. in [24] proposed energy optimized multipath protocol. They preserve energy by modifying route update information of MMRE-AODV protocol named as OMMRE-AOMDV protocol. This protocol not reduces energy consumption, it also minimize average end-to-end delay, routing overhead. Also, improves packet delivery and throughput of the network. Koffka Khan and Wayne Goodridge [25] address fault tolerance issue for Wireless Sensor Networks. The protocol proposed known as Multi Criteria AOMDV evaluated in lossy environments in node disjoint manner. A new path loss metric is included in the routing table. The main advantage of this protocol it avoids such paths facing with high packet drop or paths are very dynamic in nature. Jing Yang et al., [26] proposed a multipath routing incorporating dynamic clustering and any colony optimization. The routing protocol is designed to address energy consumption issue by nodes in the network. The cluster head are selected based on residual energy. The ACO is to find multiple paths between cluster head and destination node. Final selection of route is based on energy consumption of individual paths by cluster heads. This novel technique increases the network lifetime by reducing node energy consumption. Dhriti Sundar Maity and Subhrananda Goswami [28] use ACO for MTSP and Swarm Inspired Multipath data transmission. The congestion control issue in MANET is address nicely. In the proposed protocols paths are continuously get updated using path pheromone scents. The congestion measures using total queue length and hop distance. The data delivery through congested route is avoided. S. Saqaeeyan and M. Roshanzadeh [29] reliability and load balancing issues are considered in a wireless sensor network. This protocol also ensures that data delivery to the destination some extend guaranteed. 
The routing selection is based on remaining energy of nodes within the transmission of any sender node. The simulations results show that reliability is improved and it helps in increasing overall network life time.

\section{SYSTEM AND NETWORK MODEL}

Link disjoint paths are the path between source and destination pair where no link is common between source and destination node $[6,7]$.

Node disjoint paths are path between source and destination pair where no node is common except source and destination [6,7]. The node disjoint path is always link disjoints in nature because in node disjoint path no link is common between source and destination node. But link disjoint not necessarily node joint because node may be shared by various link. In fig. 1 three different link disjoint paths shown between $S$ (source) and $D$ (Destination) node are $S-a-b-c-d-D, \mathrm{~S}-i-k-e-g-D$ and S-me-f-D. The last two paths share the node e. In Fig. 2 there are two node disjoint paths shown between $S$ and $D$ node are $S-a-b-c-d-D$ and $S-e-f-g-h-D$ does not share any node.

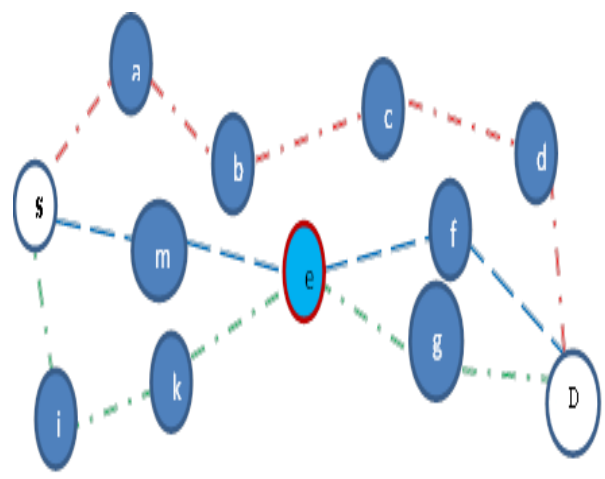

Fig.1. Link Disjoint Path

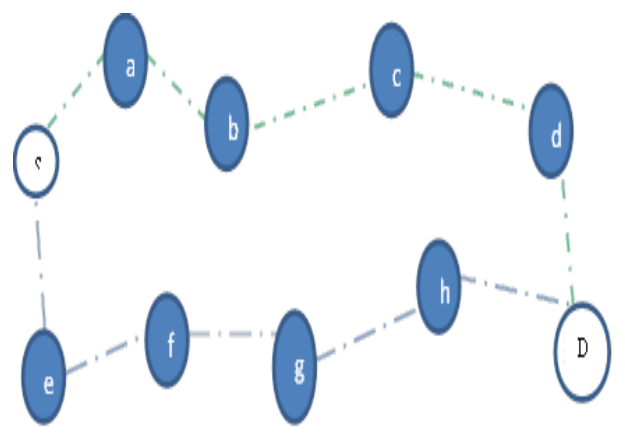

Fig.2. Node Disjoint Path

\section{A. Ad Hoc on Demand Multipath Distance Vector $(A O M D V)$}

Ad Hoc on Demand Multipath Distance Vector (AOMDV) [2, 3, 4, 5] protocol is a multipath variation of AODV protocol. The main objective of this protocol is to achieve efficient fault tolerance i.e. quick recovery from route failure. The protocol computes multiple links disjoint loop free paths per route discovery. If one path fails the protocol switches to other available paths. However, only link disjoint routes are selected (node disjoint routes are also link disjoint). The destination node replies with only $\mathrm{k}$ copies out of many link-disjoint paths, i.e. Route Request (RREQ) packets arrive through unique neighbors and nodes apart from the first hop are replied. Further, to avoid loop 'advertised hop count' is used in the routing table of nodes. The protocol only accepts alternate route with hop count less than the advertised hop count. A node can receive a routing update via a RREQ or Route Reply (RREP) packet either forming or updating a forward or reverse path. Routing updates received via RREQ and RREP are routing advertisement messages.

\section{Simulation SETUP AND RESUlts ANALYSIS}

We carried out the simulation works on NS-2.34 [18]. The table 1 shows different simulation parameters and Table 2 shows the different simulation parameters values considered for the simulation work. Here, we varied the number of nodes and node pause time. The Random Waypoint Mobility model is considered for node movement. This model is widely accepted and used in simulation of ad hoc network as well sensor networks. In this model node randomly chooses new destination and move towards it. Node moves towards destination with velocity between 0 to max. Further, node stay at particular location for certain time period known as pause time. This means a time epoch for which a node will stay in particular location. After expire of time epoch nodes are moving towards new destination within the simulation area [19].This process continue until simulation time. For the performance analysis we have computed average endto-end delay, routing overhead and throughput. The results are computed from several simulation runs. The results are analyzed using Matlab [20] and awk programming.

Table 1. Simulation Parameters

\begin{tabular}{|l|l|}
\hline \multicolumn{1}{|c|}{ Parameter } & \multicolumn{1}{c|}{ Specifications } \\
\hline MAC Protocol & IEEE 802.11 DCF \\
\hline Radio Propagation Model & Two-ray ground reflection model \\
\hline Channel type & Wireless channel \\
\hline Mobility Model & Random Way Point \\
\hline Antenna model & Omni-directional \\
\hline Traffic Source & CBR \\
\hline Routing Protocol & $\begin{array}{l}\text { Node Disjoint AOMDV } \\
\text { Link Disjoint AOMDV }\end{array}$ \\
\hline
\end{tabular}

Table 2. Values of Simulation Parameters

\begin{tabular}{|l|l|}
\hline \multicolumn{1}{|c|}{ Parameter } & \multicolumn{1}{c|}{ Values } \\
\hline Simulation Time & $1000 \mathrm{~s}$ \\
\hline Simulation Area $(\mathrm{X} * \mathrm{Y})$ & $1500 \mathrm{~m} \times 1500 \mathrm{~m}$ \\
\hline Packet Size & 512 bytes \\
\hline Packet Rate & 4 packets/sec. \\
\hline Transmission Range & $250 \mathrm{~m}$ \\
\hline Bandwidth & $2 \mathrm{Mbps}$ \\
\hline No. of Nodes & $10,20,30,40,50,100$ \\
\hline Pause time & $2,5,10,15$ \\
\hline Max. speed of nodes $(\mathrm{m} / \mathrm{s})$ & 10 \\
\hline
\end{tabular}




\section{A. Throughput}

Fig. 3 shows the throughput of Node Disjoint AOMDV with varying node density and node pause time. Throughput gradually increases with increasing node pause time and number of nodes till 40 nodes. The throughput is almost become stable at 40 nodes onwards till 100 nodes when pause time is $15 \mathrm{~s}$. The overall throughput is good in pause time $2 \mathrm{~s}$. The maximum achievable throughput is $160.43 \mathrm{kbps}$.

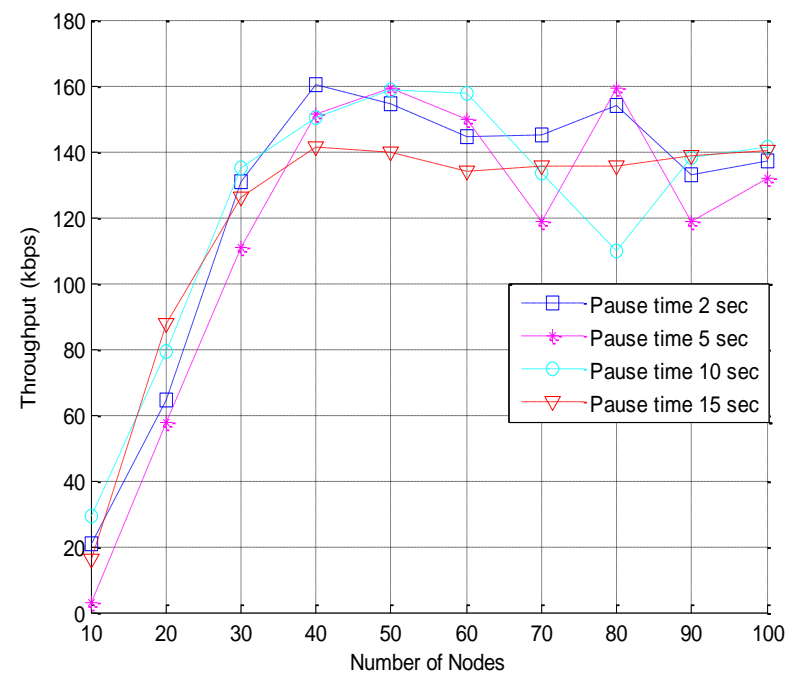

Fig.3. Throughput of Node Disjoint Multipath

Fig.4 shows the throughput of Link Disjoint AOMDV with varying node density and pause time. Throughput gradually increases with increasing number of nodes till 40 nodes and node pause time similar to Node Disjoint AOMDV. The throughput is decreases 40 nodes onwards in all pause time till 70 nodes. At 80 nodes slight increases in throughput is noticed except node pause time 10s. The maximum achievable throughput is $165.39 \mathrm{kbps}$.

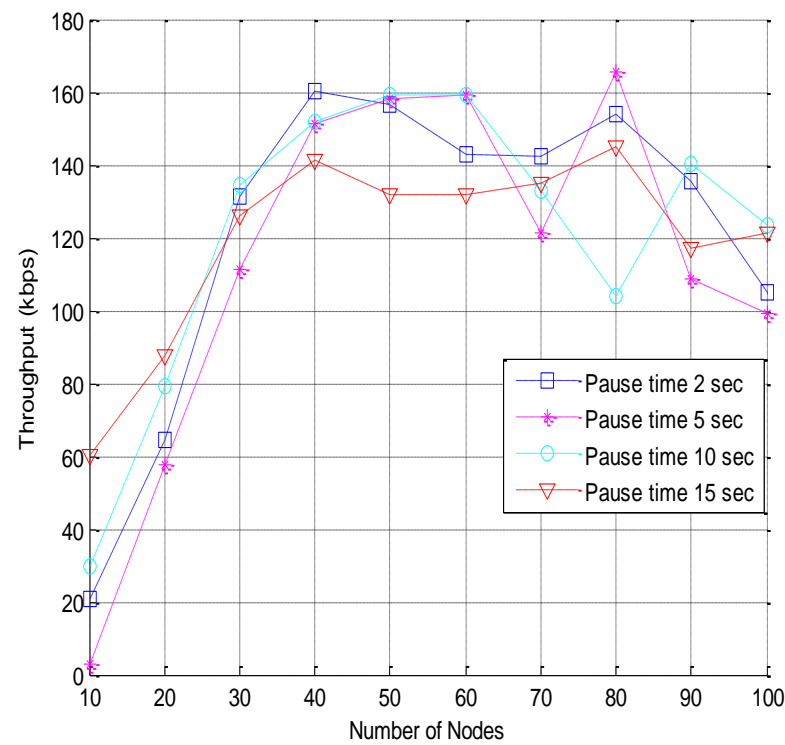

Fig.4. Throughput of Link Disjoint Multipath

\section{B. End-to-End delay}

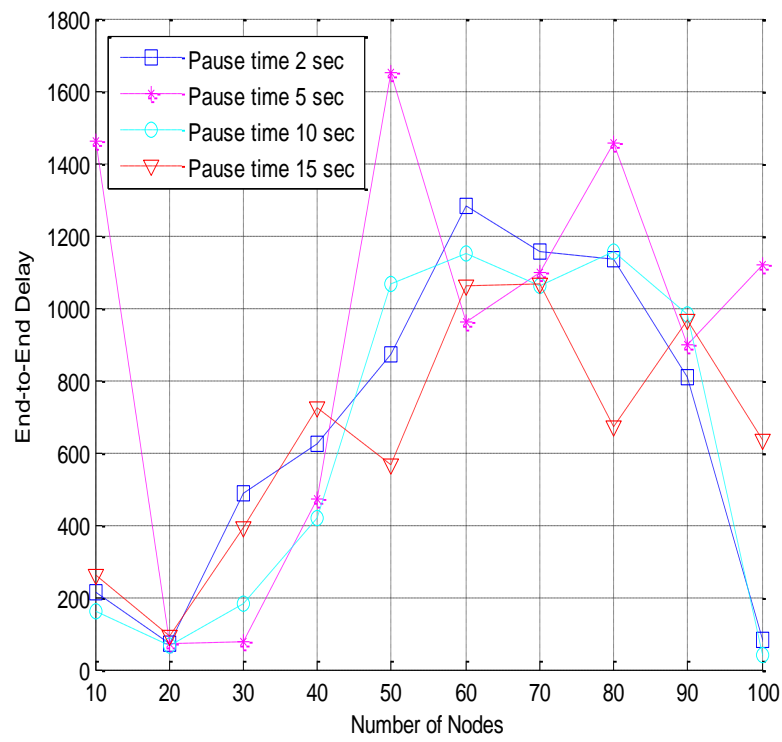

Fig.5. End-to-End delay Node Disjoint Multipath

Fig.5 shows the end-to-end delay of Node Disjoint AOMDV with varying node density and node pause time. When number of node moves from 10 to 20 for all pause time end-to-end delay is decreases, and afterward delay gradually increases with increasing number of nodes till 40 nodes and node pause time. Delay is further decreases 90 nodes onwards except at pause time $5 \mathrm{~s}$. The delay at pause time $2 \mathrm{~s}$ and $10 \mathrm{~s}$ achieves the minimum delay for number of node is 100 .

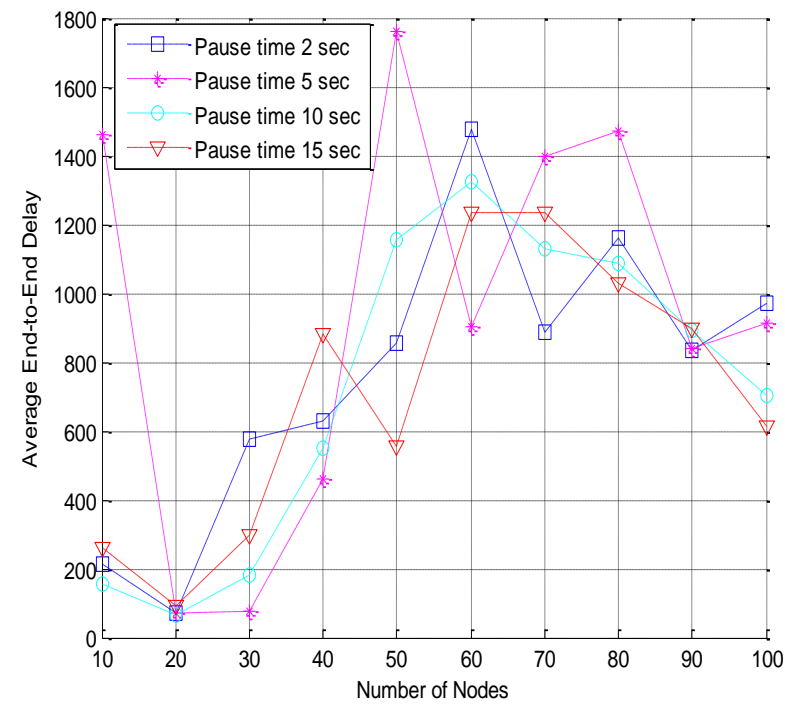

Fig.6. End-to-End delay Link Disjoint Multipath

Fig.6 shows the End-to-End delay of Link Disjoint AOMDV with varying node density and node pause time. When number of node moves 10 to 20 for all pause time end-to-end delay is decreases, and afterward delay gradually increases with increasing number of nodes till 50 nodes and node pause time except pause time $15 \mathrm{~s}$. Except at node pause time 5 s delay increases when nodes 
move from 50 to 60 . From 50 nodes onwards gradually delay decreases till 100 nodes except at pause time $2 \mathrm{~s}$ and $5 \mathrm{~s}$. The delay at pause time $10 \mathrm{~s}$ achieves the minimum delay i.e. 66.6179 for number of node is 20 .

\section{Routing Overhead}

Routing overhead gives count of total number of routing packets generated during simulation by routing protocol.

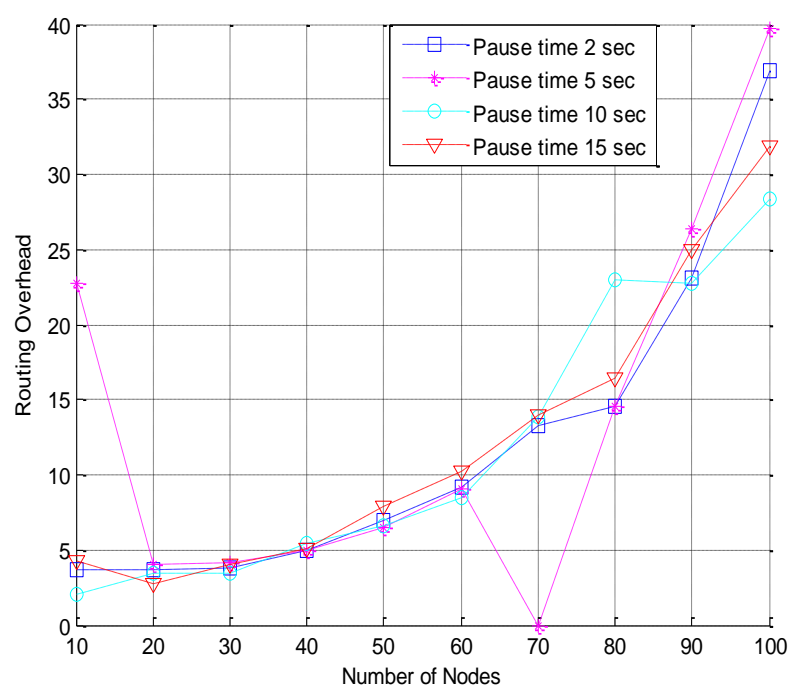

Fig.7. Routing Overhead Node Disjoint Multipath

Fig.7 shows the routing overhead of Node Disjoint AOMDV with varying node density and node pause time. At pause time $5 \mathrm{~s}$ for number of node 70 noticed minimum routing overhead. But routing overhead is gradually increases with increasing number of nodes and node pause time. The overall routing overhead is good when node pause time is $10 \mathrm{~s}$. The routing overhead is maximum at node pause time 5 s for number of nodes 100 .

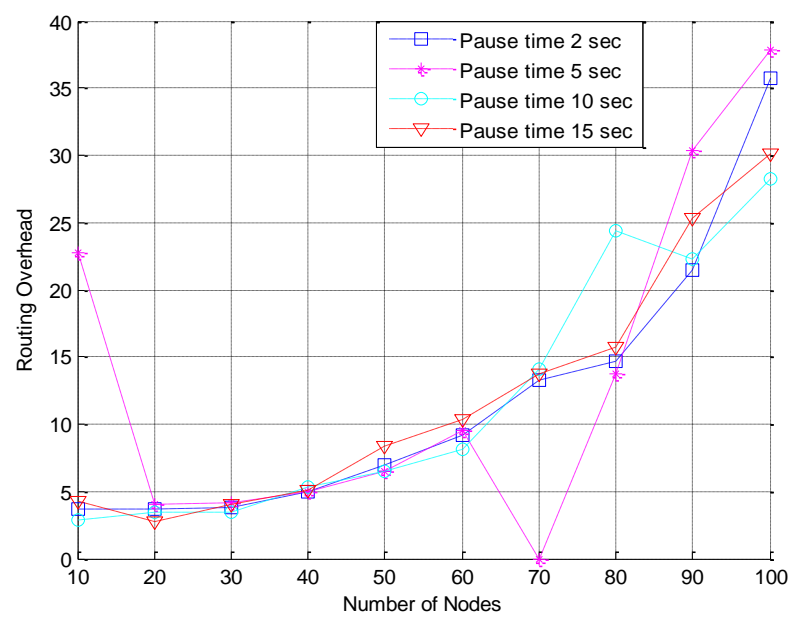

Fig.8. Routing Overhead Link Disjoint Multipath

Fig.8 shows the routing overhead of Link Disjoint AOMDV with varying node density and node pause time. Similar to node disjoint with pause time 5 s for 70 nodes noticed minimum routing overhead. But routing overhead is gradually increases with increasing number of nodes and node pause time. The overall routing overhead is good when node pause time is $2 \mathrm{~s}$. The routing overhead is maximum at node pause time $5 \mathrm{~s}$ for number of nodes 100 .

\section{CONCLUSION AND FUTURE WORKS}

We have evaluated the performance between Link Disjoint and Node Disjoint Multipath variant of AOMDV protocol. These protocols investigated with varying node density and pause time. Pause time play very vital role on the performance of routing protocols. If pause time increases gradually it seems the network become more static and connectivity among nodes is more stable. The small pause time indicate dynamic network. Various QoS matrices are computed to analyze the performance. It is evident from the results that node disjoint multipath performs better as compare to link disjoint multipath routing technique. Theoretically, also we have noticed that node disjoint is better compare to link disjoint and simulation results also justified the same. We have only considered random way point mobility model for this analysis. In future, Link Disjoint and Node Disjoint Multipath AOMDV protocols may be investigate with other node mobility models and other network scenarios for better insight understanding.

\section{REFERENCES}

[1] C. Siva Rama Murthy and B.S Manoj, "Ad Hoc Wireless Networks Architectures and Protocols", Second Edition, Pearson, 2008.

[2] Jiazi Yi, AsmaaAdnane, Sylvain David, and Benoît Parrein, "Multipath optimized link state routing for mobile ad hoc networks", Ad Hoc Networks, Vol. 9, No.1, pp. 28-47, 2011.

[3] Tsai, J., \& Moors, T., "A review of multipath routing protocols: from wireless ad hoc to mesh networks", In Proceedings of ACoRN early career researcher workshop on wireless multihop networking, Sydney, July 17-18, 2006.

[4] M. K. Marina and S. R. Das, "Ad-hoc on-demand multipath distance vector routing", Wireless Communication Mobile Computing, Vol. 6, No. 7, pp. 969-988, Nov. 2006.

[5] S. Das, C. Perkins and E. Royer, "Ad Hoc On-Demand Distance Vector (AODV). Routing”, IETF RFC3561, July 2003.

[6] Yuchun Guo, Fernando Kuipers and Piet Van Mieghem, "Link-Disjoint Paths for Reliable QoS Routing", International Journal of Communication Systems, Vol. 16 No.9,2003, pp. 779-798.

[7] M. Radi et al., "Multipath Routing in Wireless Sensor Networks: Survey and Research Challenges", Sensors 2012, Vol. 12, 2012, pp.650-685.

[8] Kejia Zhang, Guisheng Yin, Qilong Han, and Junyu Lin, "DFDP: A Distributed Algorithm for Finding Disjoint Paths in Wireless Sensor Networks with Correctness Guarantee, International Journal of Distributed Sensor Networks, Vol., 2014, pp. 1-11.

[9] Natarajan Meghanathan, "Performance Comparison of Link, Node and Zone Disjoint Multi-Path Routing Strategies and Minimum Hop Single Path Routing for Mobile Ad Hoc Networks", International Journal of 
Wireless \& Mobile Networks (IJWMN) Vol.2, No.4, November 2010, pp.13-19.

[10] Xiaoxia Huang and Yuguang Fang, "Performance Study of Node-Disjoint Multipath Routing in Vehicular Ad Hoc Networks", IEEE Transactions on Vehicular Technology, Vol. 58, No. 4, 2009, pp.1942-1950.

[11] Wesam Almobaideen, Roba Al-Soub, Azzam Sleit, "MSDM: Maximally Spatial Disjoint Multipath Routing Protocol for MANET", Communications and Network, Vol.5, No.4, 2013, pp. 316-322.

[12] Abhishek Bande and Gaurav Deshmukh, "Node Disjoint Multipath Routing Approach for Controlling Congestion in Manets", Global Journal of Computer Science and Technology Network, Web \& Security, Vol.12, Issues. 17, 2012, pp.39-45.

[13] Sonia Waharte and Raouf Boutaba, "Totally Disjoint Multipath Routing in Multi-hop Wireless Networks", In the proceeding of IEEE International Conference on Communications, Vol.9, 2006,pp.5576-5581.

[14] Vidya Kudachi and Vinod Jadhav, "Performance Evaluation of Node Disjoint Multipath Routing Protocol based on AODV for MANETs", International Journal of Advanced Electrical and Electronics Engineering (IJAEEE), Vol.2, Issue-6, 2013, pp.2278-8948.

[15] M.Nagaratna et al. "Computation of Multiple Paths in MANETs Using Node Disjoint Method", IJCSI International Journal of Computer Science Issues, Vol. 8, Issue 3, No. 1, May 2011, PP.550-554.

[16] V.K. Barbudhe et al., "Node-disjoint Multipath Routing Method Based on AODV Protocol", International Journal of Research in Advent Technology, Vol.2, No.2, February 2014.

[17] Shuchita Upadhayaya and Charu Gandhi, "Node Disjoint Multipath Routing Considering Link and Node Stability protocol: A characteristic Evaluation”, IJCSI International Journal of Computer Science Issues, Vol. 7, Issue 1, No. 2, January 2010, pp.18-25.

[18] The Network Simulator. http://www.isi.edu/nsnam/ns/.

[19] Radhika Ranjan Roy, "Handbook of Mobile Ad Hoc Networks for Mobility Models", Springer, New York Dordrecht Heidelberg London, ISBN 978-1-4419-6048-1 e-ISBN 978-1-4419-6050-4.

[20] The Math Works: http://www.mathworks.com

[21] P.Periyasamy and E.Karthikeyan "Survey of Current Multipath Routing Protocols for Mobile AD Hoc Networks", I. J. Computer Network and Information Security, 2013, 12, 68-79.

[22] P.Periyasamy and E.Karthikeyan, "Performance Comparison and Evaluation of Different Multipath Routing Protocols Based on Various Scenario and Traffic Patterns for Mobile AD Hoc Networks", International Journal of Computer Network and Information Security, 2013, 1, 24-32.

[23] Koffka Khan and Wayne Goodridge, "Impact of Multipath Routing on WSN Security Attacks", I.J. Intelligent Systems and Applications, 2014, 06, 72-78.

[24] P.Periyasamy and E.Karthikeyan, "Energy Optimized Ad hoc on-Demand Multipath Routing Protocol for Mobile Ad hoc Networks", International Journal of Intelligent Systems and Applications, 2014, 11, 36-41.

[25] Koffka Khan and Wayne Goodridge, "Fault Tolerant
Multi-Criteria Multi-Path Routing in Wireless Sensor Networks", International Journal of Intelligent Systems and Applications, 2015, 06, 55-63.

[26] Jing Yang, Wei Zhao, Mai $\mathrm{Xu}$ and Baoguo $\mathrm{Xu}$, "A Multipath Routing Protocol Based on Clustering and Ant Colony Optimization for Wireless Sensor Networks", International Journal Computer Network and Information Security, 2009, 1, 49-59.

[27] Dhriti Sundar Maity and Subhrananda Goswami, "Multipath Data Transmission with minimization of Congestion Using Ant Colony Optimization for MTSP and Total Queue Length", International Journal Computer Network and Information Security, 2015, 3, 26-34.

[28] S. Saqaeeyan and M. Roshanzadeh, "Improved Multi-Path and Multi-Speed Routing Protocol in Wireless Sensor Networks", International Journal of Computer Network and Information Security, 2012, 2, 8-14.

\section{Authors' Profiles}

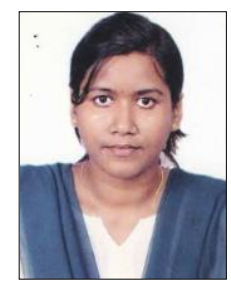

Indrani Das did her B. E. and M.Tech in Computer Science. She is working as Assistant Professor in Computer Science department in Assam University (A Central University), Assam, India. She received her Ph.D from School of Computer and Systems Sciences, Jawaharlal Nehru University, New Delhi, India in 2015. Her current research interest includes Mobile Ad hoc Networks and Vehicular Ad hoc Networks..

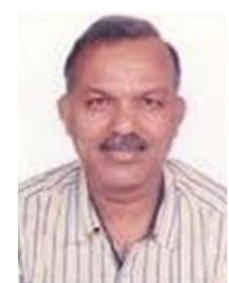

D. K. Lobiyal received his Ph.D and $M$. Tech (Computer Science) from School of Computer and Systems Sciences, Jawaharlal Nehru University, New Delhi, India in 1996 and 1991, respectively, and B. Tech. (Computer Science and Engineering) from Lucknow University, India in 1988. He is currently working as Professor at School of Computer and Systems Sciences, Jawaharlal Nehru University, New Delhi, India.

His research interest includes Mobile Ad hoc Networks, VoD Bioinformatics and Natural Language Processing. Dr. Lobiyal has published papers in International journals and conferences including IEEE, Wiley \& Sons, Springer, Inder Science, WSEAS, IGI Global, ACTA Press, and ACM.

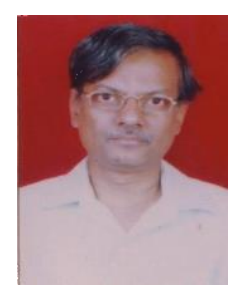

C. P. Katti is a Professor in the School of Computer and Systems Sciences, Jawaharlal Nehru University, India. He received degree of M.S. in Applied Mathematics from University of Missouri, Columbia, MO., USA in 1976 and awarded Ph.D in Scientific Computation/ Numerical Analysis from IIT Delhi in 1981. He is major in parallel processing and scientific computing. He published more than 30 papers in international journals of repute.

How to cite this paper: Indrani Das, D.K. Lobiyal, C.P.Katti,"An Analysis of Link Disjoint and Node Disjoint Multipath Routing for Mobile Ad Hoc Network", International Journal of Computer Network and Information Security(IJCNIS), Vol.8, No.3, pp.52-57, 2016.DOI: 10.5815/ijcnis.2016.03.07 